\title{
Análise cienciométrica sobre a relação da vivência de eventos de vida produtores de estresse e desenvolvimento de câncer
}

\author{
scientometrical analysis on the relationhsip between experience \\ stress-producing life events and cancer
}

Fernanda Paini Leite, ${ }^{1}$ Bruna Alexandra da Silva Cruz,, Marcelo Picinin Bernuci, ${ }^{1}$ Mirian Ueda Yamaguchi $^{1}$ 'Centro Universitário Cesumar, Maringá, PR, Brasil.

Recebido em: 10/08/2016 / Aceito em: 15/09/2016 / Publicado em: 30/09/2016

fernanda.leite@unicesumar.edu.br

\section{RESUMO}

Objetivo: identificar a produção científica nacional e internacional sobre a possível associação da vivência de eventos de vida produtores de estresse e o desenvolvimento de câncer. Métodos: estudo cienciométrico sobre a produção científica veiculada em periódicos indexados nos bancos de dados United States National Library of Medicine (PubMed), Scientific Eletronic Library Online (SciELO) e trabalhos disponíveis na Biblioteca Virtual em Saúde (BVS) por meio de busca pelos seguintes descritores; "life events influence cancer", "stressful life events cancer" e "traumatic events cancer" e seus correspondentes em língua portuguesa. Resultados: foram selecionados 82 artigos, de um total de 2.176. Verificou-se crescente número de publicações a partir da década de 90, destacando maior parte dos autores da América do Norte. Observou-se maior associação dos eventos estressores com o câncer de mama e a predominância de amostragem do grupo feminino. O luto, perda e a frustração destacaram-se como eventos estressores relacionados à maior possibilidade de desenvolvimento de câncer. Dos estudos selecionados $40,9 \%$ apresentaram correlação positiva, $25,3 \%$ correlação negativa e 33,8\% foram inconclusivos. Considerações finais: verificou-se controvérsia nos resultados das publicações científicas no que tange à análise de correlação entre o desenvolvimento de câncer e presença de eventos de vida produtores de estresse, sugere-se a necessidade da realização de novos estudos que abordem esta temática.

Palavras-chave: Trauma; Fatores de Estresse; Câncer.

\section{ABSTRACT}

Objective: to identify the national and international scientific literature on the possible association of experience stressful life events and the development of cancer. Methods: scientometrical study of the scientific production published in indexed journals in databases United States National Library of Medicine (PubMed), Scientific Electronic Library Online (SciELO) and the works available on the Virtual Health Library (VHL) through search by following descriptors; "Life events influence cancer", "stressful life events cancer" and "traumatic events cancer" and their counterparts in Portuguese. Results: 82 items were selected for evaluation (of a total of 2,176). There are a growing number of publications from the 90s, highlighting most of the authors of North America. We observe a higher association of stressful events with breast cancer and sampling predominance of the female group. Grief, loss and frustration stood out as stressful events related to the greater possibility of developing cancer. $40.9 \%$ of the selected studies showed positive correlation, $25.3 \%$ negative correlation and $33.8 \%$ were inconclusive. Closing remarks: there was controversy in the results of the scientific literature, we found positive and negative correlations of stress with cancer, as well as inconclusive results. It suggests the need for more studies on this topic.

Keywords: Trauma; Stressor Factors; Cancer. 


\section{INTRODUÇÃO}

Segundo a Organização Mundial da Saúde (OMS), é esperado, nas próximas décadas um impacto relacionado ao câncer nos países em desenvolvimento. Estima-se que, para esses países, o câncer na população corresponda a $80 \%$ dos mais de 20 milhões de casos novos estimados para 2025 .

As causas conhecidas para o câncer são apresentadas de forma multifatorial, ou seja, existem diversos fatores que podem influenciar no desenvolvimento desta alteração, tais como estilo de vida, exposição excessiva à radiação, fatores hormonais, fatores hereditários, mutações genéticas e fatores psicológicos, como o estresse. ${ }^{2} \mathrm{O}$ estresse causado por eventos diários vividos, bem como os eventos incomuns, como perda de uma pessoa próxima repentinamente ou doenças graves em si mesmo, podem desencadear, em algumas pessoas, a sensação de incapacidade de enfrentar e resolver a situação em questão. ${ }^{3}$

O estresse tem um papel importante na etiologia de diversas patologias, de forma que os fatores psicológicos afetam o sistema imune, deprimindo as condições do mesmo e aumentando os riscos de desencadear diversas doenças. ${ }^{4,5}$ As condições psicológicas estão inteiramente ligadas à liberação de hormônios como o cortisol. Quando expostos ao estresse ocorre a ativação crônica e repetida do eixo hipotálamo-hipófise-adrenal, que pode resultar em alterações patológicas, como as neoplasias. ${ }^{6,7}$ A secreção crônica de cortisol, em resposta a eventos estressores, tem sido associada com o desenvolvimento de patologias diversas como: doença cardíaca coronoriana, ${ }^{4}$ osteoporose, ${ }^{8}$ úlcera de membros inferiores ${ }^{9}$ e recentemente há um crescente número de estudos que apontam uma correlação positiva entre aumentos crônicos dos níveis plasmáticos de cortisol induzido por estresse e desenvolvimento de neoplasias..$^{10-13}$

Tentativas de associar a etiologia das neoplasias malignas com os eventos estressantes diários datam desde a Grécia antiga, onde Galeno sugeria que muIheres melancólicas e depressivas eram mais propensas a desenvolver o câncer do que aquelas com um alto astral. ${ }^{6}$ Embora até então muitos estudos tenham sido realizados neste âmbito, ainda não há um consenso quanto à influência dos eventos estressores diários no desenvolvimento de neoplasias. Dessa forma, analisamos no presente estudo, a produção científica nacional e internacional direcionada a investigar a possível relação entre estresse e câncer. A análise cienciométrica desse tema torna-se relevante uma vez que os modos de vida modernos têm desencadeado frequentes distúrbios psicossociais associados a eventos estressantes, bem como aumento progressivo de doenças crônicas não comunicáveis como o câncer. ${ }^{7,10,11}$

\section{MATERIAIS E MÉTODO}

Foi realizado estudo cienciométrico da literatura científica nacional e internacional sobre a correlação entre estresse e desenvolvimento de câncer. $O$ objeto da análise foi identificar a produção científica veiculada em periódicos indexados nos bancos de dados United States National Library of Medicine (PubMed), Scienti- fic Eletronic Library Online (SciELO) além de trabalhos disponíveis na Biblioteca Virtual em Saúde (BVS).

A busca por artigos científicos foi realizada nos meses de março e abril de 2016. Para isso foram utilizados os seguintes descritores: "life events influence cancer", "stressful life events cancer" e "traumatic events cancer" e seus correspondentes em língua portuguesa "eventos de vida influentes no câncer", "eventos de vida estressores e câncer" e "eventos traumáticos no câncer", respectivamente. Em um primeiro momento a busca identificou 2.176 artigos científicos, dos quais 688 estavam relacionados com o descritor "life events influence cancer", 585 com "stressful life events cancer" e 903 com o descritor "traumatic events cancer". Foram excluídos trabalhos cujos descritores foram empregados em temáticas não relacionadas ao tema central desta pesquisa, como exemplo, estudos sobre a relação do estresse pós-traumático em pacientes já diagnosticados com câncer ou ainda a utilização do termo relacionado ao estresse oxidativo sofrido pela célula, durante o desenvolvimento da doença, sendo ainda excluídos artigos encontrados em duplicidade, nas bases de dados utilizadas nesta pesquisa.

Após análise crítica dos títulos e resumos, 82 artigos atenderam aos critérios de inclusão e os mesmos foram classificados segundo o ano de publicação do artigo científico, o local de origem do autor principal do trabalho e o tipo de estudo utilizado, além da identificação do evento estressor e o câncer mais frequente. Esses dados foram tabulados e organizados em planilhas do programa Microsoft Excel 2010.

\section{RESULTADOS}

O estudo cienciométrico sobre eventos estressores e câncer identificou artigos publicados na base de dados SciELO, PubMed e Bireme, no período compreendido entre os anos de 1970 e 2015. Os mesmos foram agrupados por quinquênios, a partir do qual observa-se aumento no número de publicações científicas, a partir do início da década de 90, atingindo maior volume de pesquisas no quinquênio 2006-2010 conforme a figura 1 .

Na figura 2, é possível identificar a origem dos pesquisadores que publicaram artigos científicos relacionados ao tema, apontando maioria proveniente da América do Norte, correspondendo a 25(30,48\%) publicações, seguido da Europa Ocidental 20 (24,39\%), Europa do Norte 11 (13,41\%), Ásia 10 (12,19\%), Europa Central 9 (10,97\%), Europa do Sul, Europa Oriental e Oceania $2(7,29 \%)$, e com apenas $1(1,21 \%)$ publicação de autores provenientes da América do Sul.

Dentre os 82 artigos analisados, foram identificados cinco modalidades de pesquisa, das quais 28 $(34,1 \%)$ são trabalhos de revisão sistemática e metanálise, $23(28,0 \%)$ estudos de coorte retrospectivos, 21 $(25,6 \%)$ estudos de caso-controle. Apenas 6 (7,31\%) artigos são estudos transversais observacionais e 4 $(4,87 \%)$ estudos de coorte prospectivo (Figura 3 ).

Considerando o critério de estratificação Qualis na área interdisciplinar, instituído pela Coordenação de Aperfeiçoamento de Pessoal de Nível Superior (CAPES) e fundação do Ministério de Educação e Cultura (MEC), dos 82 artigos, a maioria procede de periódicos científi- 


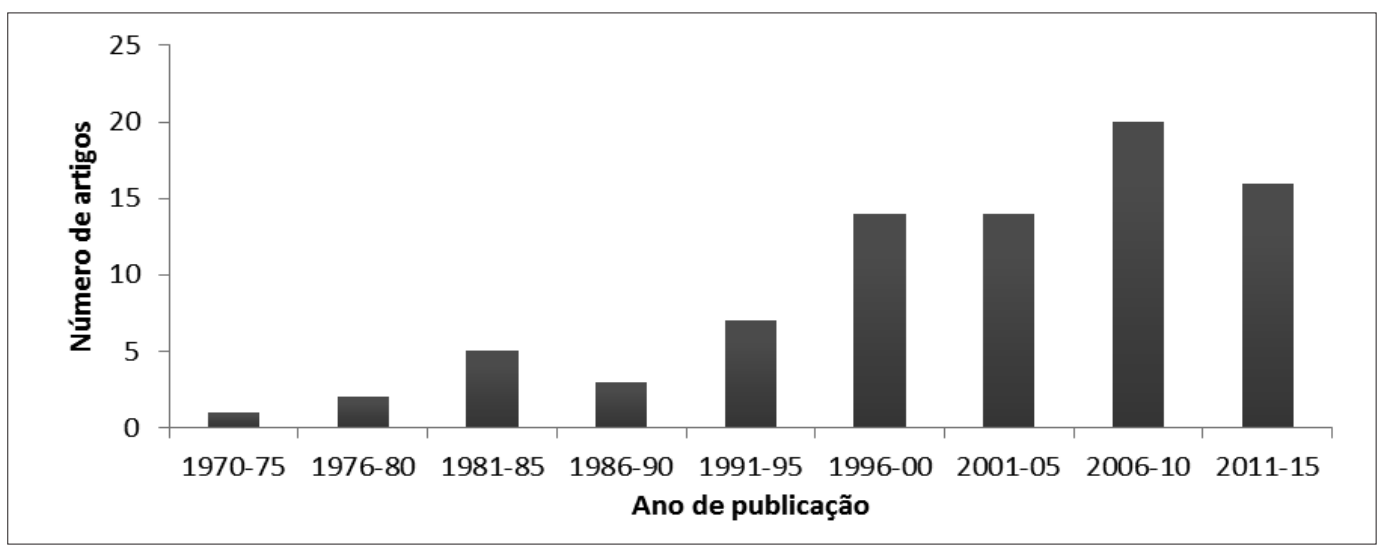

Figura 1 - Número de artigos publicados nas bases de dados SciELO, PubMed e Bireme segundo o ano de publicação.

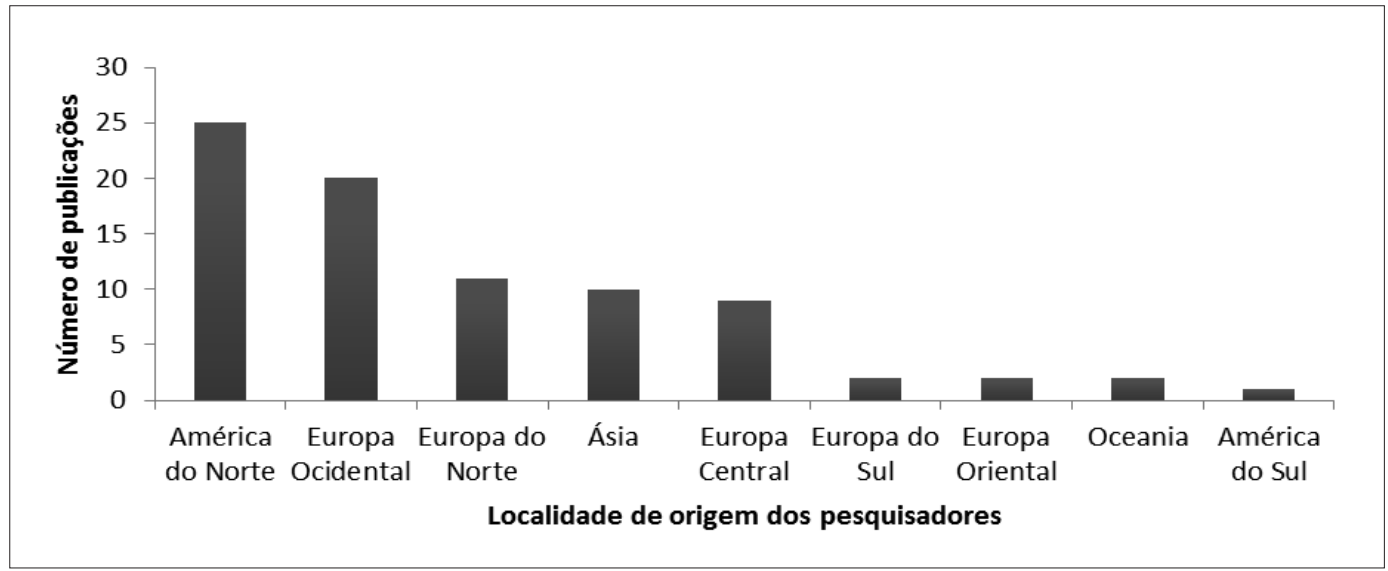

Figura 2 - Número de artigos publicados classificados segundo o continente de origem do autor principal dos estudos.

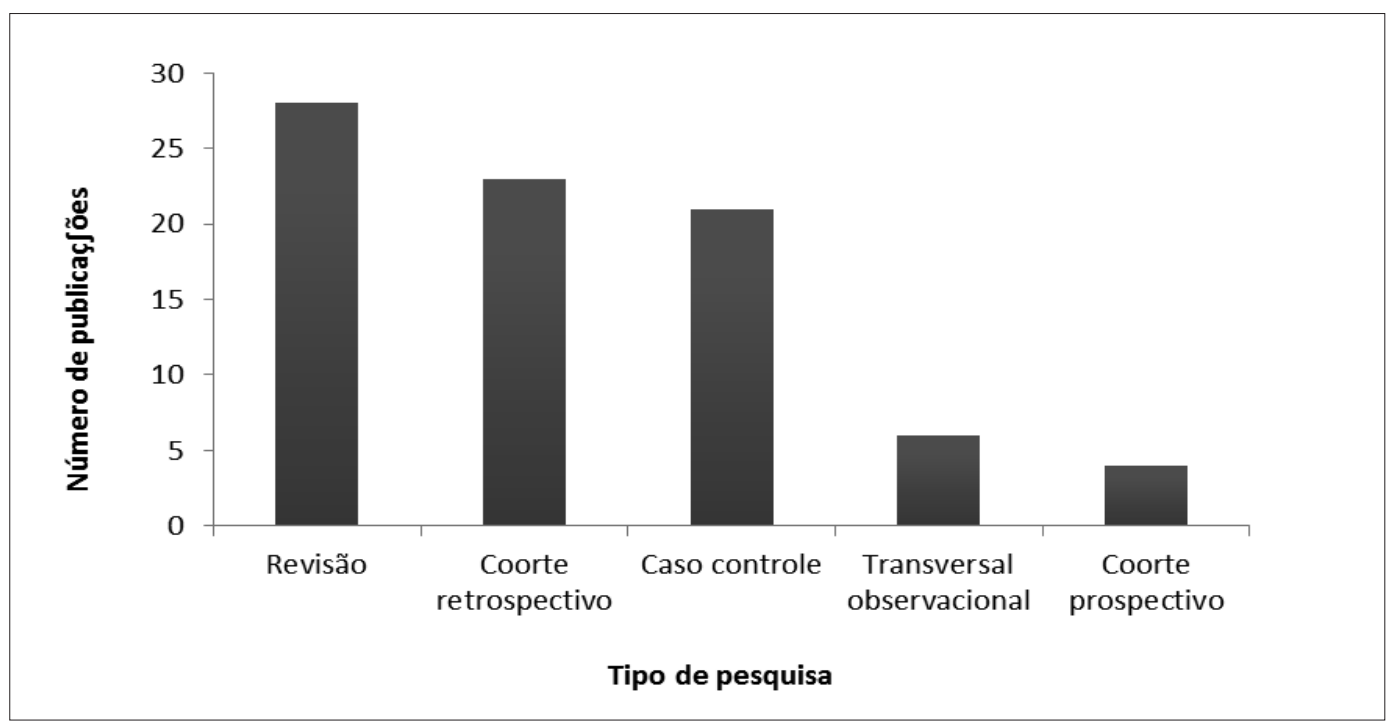

Figura 3 - Número de artigos classificados de acordo com o tipo de pesquisa.

cos classificados como os melhores estratos. A presente pesquisa identificou 38 artigos publicados em periódicos Qualis A1, seguida por 20 artigos com Qualis A2. As publicações em periódicos com Qualis B1 correspondem a um total de 9, seguido de 13 artigos no estrato Qualis B2 e 2, com classificação B3 (dados não mostrados).

Quando associada à vivência de eventos estres- sores ao desenvolvimento de câncer, 9 tipos de neoplasias foram citadas e correlacionadas positivas ou negativamente ao estresse. A relação com o câncer de mama foi descrita em $47(57,3 \%)$ artigos, em $21(25,6 \%)$ a relação envolve o câncer em geral não sendo especificada apenas uma região. Quanto ao câncer colorretal, o melanoma, câncer de colo uterino e pulmão foram 
citados em $3(3,65 \%)$ artigos. A relação do estresse com câncer cerebral, prostático e gástrico foi citado em apenas $1(1,21 \%)$ dos trabalhos. As relações entre as neoplasias citadas e sua influência pelo estresse tiveram resultados considerados positivos, negativos ou ainda associações dadas como inconclusiva pelos pesquisadores (Tabela 1).

Tabela 1 - Número de artigos publicados classificados de acordo com a correlação entre o tipo de câncer e os eventos estressores.

\begin{tabular}{lccc}
\hline \multirow{2}{*}{ Tipo de câncer } & \multicolumn{3}{c}{ Correlação entre eventos estressores e câncer } \\
\cline { 2 - 4 } & $\begin{array}{c}\text { Correlação } \\
\mathbf{n}(\%)\end{array}$ & $\begin{array}{c}\text { Sem correlação } \\
\mathbf{n}(\%)\end{array}$ & $\begin{array}{c}\text { Correlação } \\
\text { inconclusiva } \\
\mathbf{n}(\%)\end{array}$ \\
\hline Mama & $18(53,0)$ & $14(66,7)$ & $15(53,6)$ \\
Geral & $9(26,5)$ & $5(23,7)$ & $7(25,0)$ \\
Colorretal & $2(5,9)$ & $1(4,8)$ & $0(0,0)$ \\
Melanoma & $0(0,0)$ & $0(0,0)$ & $3(10,6)$ \\
Pulmão & $2(5,9)$ & $0(0,0)$ & $1(3,6)$ \\
Útero & $1(2,9)$ & $1(4,8)$ & $1(3,6)$ \\
Cérebro & $0(0,0)$ & $0(0,0)$ & $1(3,6)$ \\
Próstata & $1(2,9)$ & $0(0,0)$ & $0(0,0)$ \\
Estômago & $1(2,9)$ & $0(0,0)$ & $0(0,0)$ \\
Total & $\mathbf{3 4}(100)$ & $\mathbf{2 1}(100)$ & $\mathbf{2 8}(100)$ \\
\hline
\end{tabular}

A Figura 4 apresenta a classificação das publicações segundo o tipo de evento estressor e a correlação com o câncer. A classificação foi realizada com base no estudo de Lewis (1988), que diferencia os eventos estressores de acordo com a sua significação psicológica. ${ }^{7}$ Dentre os artigos selecionados são relatados 119 eventos de vida estressores sendo que 38 (31,9\%) publicações relacionam com estresse em geral, sem especificar o tipo; $33(32,7 \%)$ relatam estressor referente à perda ou luto, $18(15,1 \%)$ estressor relacionado à frustração, 17 (14,2\%) ameaça, 11 (9,24\%) performance, $2(1,68 \%)$ tédio e nenhum artigo relata estresse físico.

\section{DISCUSSÃO}

O presente trabalho aponta a Europa, América e Ásia na liderança no número de publicações que associam estresse com câncer, totalizando 93,9\% das publicações nos periódicos científicos identificados nesta pesquisa, sendo as demais provenientes da África e Oceania. O diretor científico da Rede SciELO, afirmou durante uma conferência realizada pela Fapesp (Fundação de Amparo a Pesquisa do Estado de São Paulo) em 2013, um crescimento da internacionalização dos periódicos dos países emergentes, onde muitos periódicos científicos dessas nações começam a abrir espaço para a publicações de pesquisadores internacionais. Porém, é bastante claro que o número reduzido de publicações por estes países está relacionado à pouca aceitação em periódicos internacionais de maior destaque. Como exemplo tem-se a China que apresenta produção científica significativa, mas que muitas vezes não encontra aceitação em periódicos internacionais renomados, tendo que publicar suas pesquisas em periódicos de nações da América do Sul que apresentam menor fator impacto científico. ${ }^{16}$

$\mathrm{O}$ destaque para o alto índice de publicações na América do Norte e Europa Ocidental pode estar relacionado ao grande interesse sobre a temática em estudo, logo que os países de ambas as regiões ocupam os dez primeiros lugares no ranking de incidência de câncer no mundo, de acordo com o último levantamento realizado pela Organização Mundial da Saúde. ${ }^{16}$

De acordo ainda, com a Globocan, os três primeiros colocados no ranking de tipos de câncer mais prevalentes no mundo são ocupados pelo câncer de pulmão, seguido do câncer de mama e câncer colorretal. ${ }^{17}$ A presente pesquisa identificou o câncer de mama como o mais relacionado aos eventos estressores, embora apresentando resultados com relação e sem relação com o estresse, além de resultados inconclusivos. Os estudos sobre câncer de pulmão e colorretal apontaram maior número de relação positiva

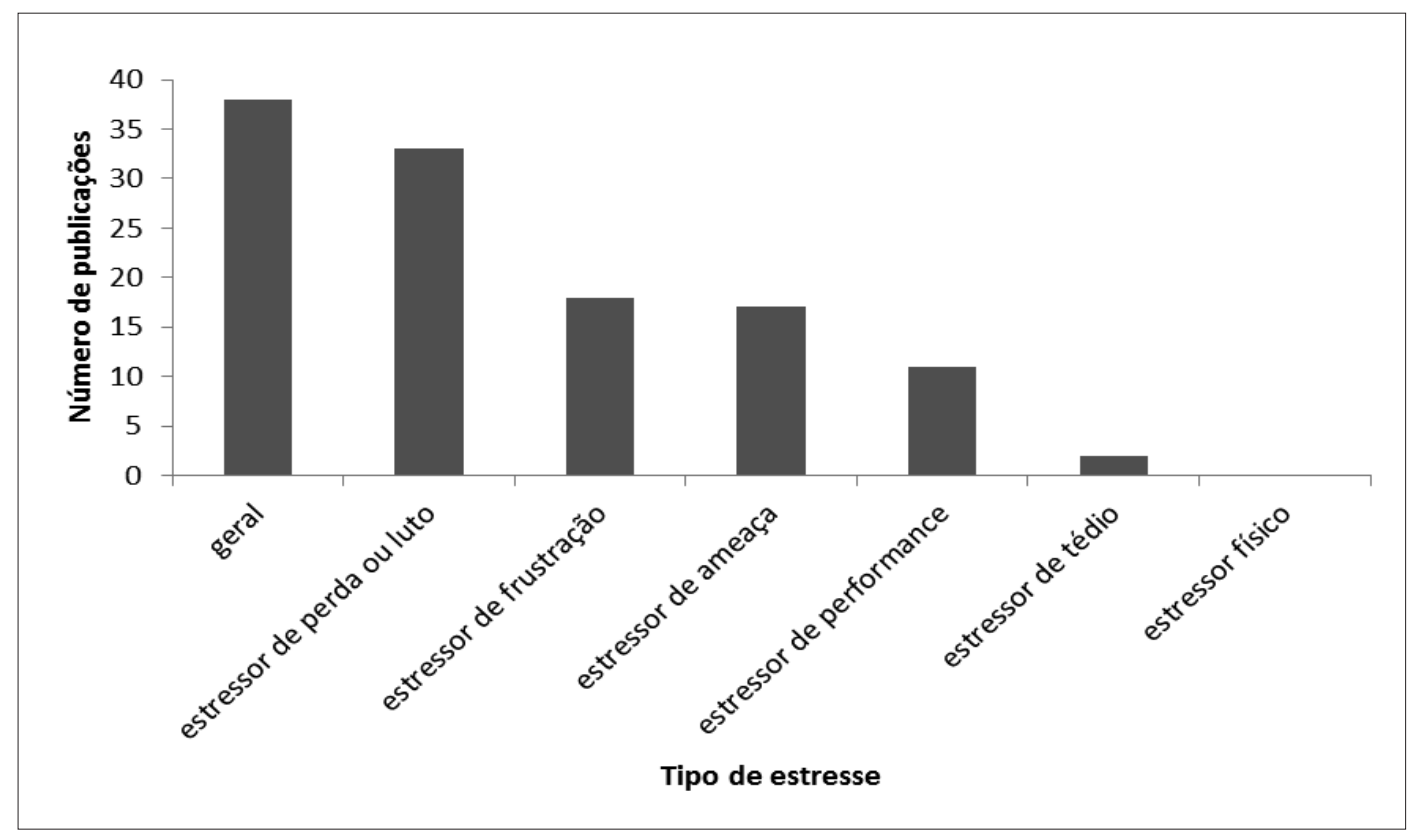

Figura 4 - Classificação de acordo com o tipo de estresse vivido pela amostra. 
com estresse. Casos de melanoma foram relatados em 3 artigos, mas apresentaram resultados inconclusivos, quando relacionados ao estresse.

As controvérsias quanto à existência de associação entre a vivência de eventos estressores e o surgimento de câncer foram nitidamente observadas. Houve equiparidade no número de publicações com resultados positivos, negativos e inconclusivos. A inconsistência nos resultados pode ser explicada pelo fato de que a maioria dos dados epidemiológicos disponíveis provém de estudos caso-controle retrospectivo; desta forma os dados obtidos dependem dos casos e controles, podendo gerar vieses de memória. Deste modo a escolha da modalidade de estudo pode ser considerada um dos fatores influentes desses resultados variados. ${ }^{18,19}$

Outra questão a considerar é a variabilidade na maneira como o estresse é conceituado, dificultando a avaliação quantitativa do papel dos fatores sociais, no aparecimento e evolução de doenças malignas. ${ }^{20}$

O número da amostra utilizada para os estudos observacionais encontrou-se bastante variável. Foram selecionados trabalhos envolvendo amostragens, inferiores a 100 participantes até pesquisas com 4 milhões de pessoas. A amostragem insuficiente foi apontada como um dos principais problemas metodológicos nos estudos abordados, fator este que torna os resultados heterogêneos e inconclusivos. ${ }^{21-23}$

As pesquisas foram realizadas com diferentes amostragens envolvendo homens e mulheres. Dentre as publicações, 5,6\% referem-se à amostragem masculina, $24,5 \%$ amostragem mista e $67,5 \%$ envolveram o público feminino. A literatura científica com maior volume de publicações voltadas ao câncer de mama justifica a maioria das pesquisas realizadas com sexo feminino, devido o câncer de mama representar o segundo tipo mais comum no mundo. ${ }^{24}$

Dentre os eventos estressores avaliados, um terço dos estudos destaca a influência do estresse de forma geral, não especificando os acontecimentos vividos pelos indivíduos portadores de câncer de forma pontual. Outra questão a considerar refere-se ao intervalo decorrente desde a vivência dos fatores estressores até o surgimento do câncer. Cerca de $50 \%$ das publicações não se referiram ao intervalo de tempo e número de eventos estressores vivenciados. Os demais estudos delimitaram os eventos estressores decorridos no período de $10(11,3 \%)$ e $5(33,9 \%)$ anos. ${ }^{25}$

A relação do câncer com o fator estressor de perda e luto foi identificado em $28,0 \%$ da amostragem total, com maior destaque para a morte do cônjuge e a perda dos pais, durante a infância. Enquanto os eventos relacionados à frustração foram relatados por $15,1 \%$ da população avaliada, com destaque para o divórcio como o evento mais representativo deste tipo de estressor. É válido ressaltar ainda que eventos estressores físicos não foram relatados, não havendo nas referidas pesquisas, associação com o desenvolvimento do câncer, embora alguns autores tenham afirmado que é possível o estresse físico desencadear condições de imunossupressão, capaz de permitir o desenvolvimento de doenças como o câncer.

Vin-Raviv ${ }^{26}$ acompanhou sobreviventes da Segunda Guerra Mundial, que passaram ao menos seis meses sob regime nazista, sofrendo condições extremas de frio e fome e relatou associação significativa aos casos positivos de câncer nesta população.

Em contrapartida, Olsen ${ }^{27}$ aponta que o aumento da incidência de câncer nos sobreviventes pós guerra, provavelmente não esteja diretamente associada à exposição aos fatores físicos, mas que possa estar indiretamente mediada pelas respostas comportamentais ao estresse psicológico sofrido, levando à utilização de métodos de escape como álcool e tabagismo (fatores de risco conhecidos para o câncer).

A explicação biológica da associação de eventos estressantes vividos com o desenvolvimento do câncer encontra-se embasado na premissa de que o estresse perturbe áreas dos sistemas neuroimunológicos e possivelmente, as funções dos eixos neuroendócrinos, levando a alterações nas concentrações sanguíneas de vários hormônios. Grande parte dos estudos não fornecem uma integração entre perpectivas psicológicas e biológicas, não havendo, portanto uma correlação satisfatória quanto a esta possível associação. ${ }^{28,29}$

\section{CONCIDERACÕES FINAIS}

A partir do estudo cienciométrico desenvolvido foi possível identificar que a América do Norte foi o continente com maior número de pesquisadores envolvidos com o tema central, tendo maior número de publicações entre os anos de 2006 e 2010.

Dentre os casos positivos para a relação estresse e câncer, o evento estressor mais relatado foi a perda de um familiar e logo, a associação do estresse ao câncer de mama foi a mais citada.

Conclui-se que falhas metodológicas como amostras pequenas e estudos que induzam os vieses de memória podem justificar os resultados controversos encontrados, assim como a variabilidade dos conceitos aplicados ao estresse e a incapacidade de isolar eventos que são específicos ao câncer, daqueles específicos para doenças em geral.

\section{AGRADECIMENTOS}

Ao Instituto Cesumar de Ciência, Tecnologia e Inovação - ICETI pela concessão dos fundos de apoio que viabilizaram esta pesquisa.

\section{REFERÊNCIAS}

1. Inca. Estimativa 2016: incidência de câncer no Brasil / Instituto Nacional de Câncer.

2. Farago PM, Ferreira DB, Reis RPJP, Gomes IP, Reis PED. My life breast cancer: report of emotional, stress. Revista de Enfermagem, UFPE 2010;4(3):1037-1042.

3. Filho JM, Moreira MD. (2010). Psicoimunologia hoje In V. A. Carvalho, M. J. Kovacs, \& M. H. P. Franco (Eds.). Temas em psico-oncologia (pp. 167-213). São Paulo: Summus.José Alencar Gomes da Silva - Rio de Janeiro: 2015.

4. Kim D, Kubzansky LD, Baccarelli A, Sparrow D, Spiro A 3rd Tarantini L, Cantone L, Vokonas P, Schwartz J. Psychological factors and DNA methylation of genes related to immune/ inflammatory system markers: the VA normative aging 
study. BMJ Open 2016;6(1):e009790. doi: 10.1136/ bmjopen-2015-009790.

5. Lasselin J, Alvarez-Salas E, Grigoleit JS. Well-being and immune response: a multi-system perspective. Curr Opin Pharmacol. 2016;29:34-41. Doi: 1016/j.coph.2016.05.003

6. Lutgendorf SK, Sood AK, Antoni MH. Host Factors and Cancer Progression: Biobehavioral Signaling Pathways and Interventions. ASCO 2010;28(26):4094-99 doi: 10.1200/ jco.2009.26.9357.

7. Moreno-smith, M; Lutgendorf, SK; Sood, AK. Impact of stress on cancer metastasis. Future Oncology 2010;6(12):1863-81. doi: 10.2217/fon.10.142

8. Azuma K, Adachi Y, Hayashi H, Kubo KY. Chronic Psychological Stress as a Risk Factor of osteoporosis. J Uoeh 2015;37(4):245-53. doi: 10.7888/juoeh.37.245.

9. Situm M, Kolić M, Spoljar S. Quality of life and psychological aspects in patients with chronic leg ulcer. Acta Med Croatica 2016;70(1):61-3.

10. Renzi C, Vadilonga V, Gandini S, Perinel G, Rotmensz N, Didier F, Rescigno M, Pravettoni G. Stress exposure in significant relationships is associated with lymph node status in breast cancer. PLoS One 2016;11(2):e0149443. doi: 10.1371/journal.pone.0149443.

11. Webster S, Chandrasekaran S, Vijayaragavan R, Sethu G. Impacto of emotional support on serum cortisol in breast cancer patients. Indian J Palloat Care 2016;22(2):141-9. doi: 10.4103/0973-1075.179607.

12. Guo X, Fu H, Kong X, Gao L, Wang W, Ma W, Yao $\mathrm{Y}$, Wang $\mathrm{R}$, Xing B. Pituicytoma coexisting with corticitroph hyperplasia: literature review with one case report. Medicine 2016;95(10):e3062. doi: 10.1097/ MD.0000000000003062.

13. Mankarious A, Dave F, Pados G, Tsolakidis D, Gidron Y, Pang $\mathrm{Y}$, Thomas $\mathrm{P}$, Hall M, Karteris $\mathrm{E}$. The pro-social neurohormone oxytocin reverses the actions of the stress hormone cortisol in human ovarian carcinoma cells in vitro. Int $\mathrm{J}$ Oncol 2016;48(5):1805-14.

14. Espindola AM. Estresse, emoções e câncer de mama: relações possíveis, Andréia Magalhães Espíndola. - 108 f. : il. [Dissertação] - Universidade Federal de Juiz de Fora, Juiz de Fora, 2012.

15. Santos VMG, Gonçalves JGF, Mussalem MM, Coelho MF. Stress: Fator Influente na Qualidade de Vida dos Professores do Ensino Fundamental. 2001. Disponível em: <http://www. ismabrasil.com.br/trabalho/17>. Acesso em: 18 jul. 2016.

16. Elton alisson (São Paulo). Fundação de Amparo à Pesquisa do Estado de São Paulo (Ed.). Revistas científicas de países emergentes aumentam processo de internacionalização. 2013. Disponível em:<http://agencia.fapesp.br/revistas _ cientificas _ de _paises_emergentes_aumentam _processo_de internacionalizacao_/18142/>. Acesso em: 15 maio 2016

17. Globocan. Section of Cancer Surveillance. Disponível em: $<$ http://globocan.iarc.fr/Pages/fact_sheets _ population. aspx>. Acesso em: 7 jul. 2016

18. Price MA, Tennant CC, Butow PN, Smith RC, Kennedy SJ, kossoff, MB and Dunn, SM: The role of psychosocial factors in the development of breast carcinoma: Part II. Life event stressors, social support, defense style, and emotional control and their interactions. Câncer 2001;91:686-697.

19. Ollonen P, Lehtonen J, eskelinen M. Stressful and Adverse Life Experiences in Patients with Breast Symptoms; a Prospective Case-control Study in Kuopio, Finland. Anticancer Research 2005;531-536.

20. Nielsen NR, Gronbaek M. Stress and breast cancer: a systematic update on the current knowledge. Nat Clin Pract Oncol 2006;612-620.

21. Schuuler, G, Schubert C. The influence of psychosocial factors on the immune system (psychoneuroimmunology) and their role for the incidence and progression of cancer. $Z$ Psychosom Med Psychother 2001;6-41.

22. Lambe M, Cerrato R, Askling J, Hsieh CC. Maternal breast cancer risk after the death of a child. Int J Cancer. Sweden 2004;763-766.

23. Schwarz S, Messerschmidt H, Doren M. Psychosocial risk factors for cancer development. Med Klin. Munich 2007;967-979.

24. Ferlay J, Soerjomataram I, Ervik M, Dikshit R, Eser S, Mathers C, Rebelo M, Parkin DM, Forman D, Bray F. GLOBOCAN 2012 v1.1, Cancer Incidence and Mortality Worldwide: IARC CancerBase No. 11 [Internet]. Lyon, France: International Agency for Research on Cancer; 2014. Available from: http://globocan.iarc.fr.

25. Schraub, S, Sancho-garnier H, Velten M. Should psychological events be considered cancer risk factors? Rev Epidemiol Sante Publique 2009;113-123.

26. Vin-Raviv N, Dekel R, Barchana M, Linn S, Keinan-Boker L. World War II-related post-traumatic stress disorder and breast cancer risk among Israeli women: a case-control study. Int Psychogeriatr 2014;6(23):499-508. doi: 10.1017/ S1041610213002081.

27. Olsen $\mathbf{M H}$, Nielsen $H$, Dalton SO, Johansen C. Cancer incidence and mortality among members of the Danish resistance movement deported to German concentration camps: 65-Year follow-up. Int J Câncer 2015;10(136):247680. doi: 10.1002/ijc. 29288.

28. Geyer S. The role of social and psychosocial factors in the development and course of cancer. Wien Klin Wochenschr 2000;112(23):986-94.

29. Eskelinen M, Norden T., Lindgren A, Wide L, Adami H. O and Holmberg L: Preoperative serum levels of follicle stimulating hormone (FSH) and prognosis in invasive breast cancer. EJSO 2004:30: 495-00.

Como citar: LEITE, Fernanda Paini et al. Análise cienciométrica sobre a relação da vivência de eventos de vida produtores de estresse e desenvolvimento de câncer. Cinergis, Santa Cruz do Sul, v. 17, n. 3, set. 2016. ISSN 2177-4005. Disponível em: <https://online.unisc.br/seer/index.php/cinergis/article/view/8133>. Acesso em: 11 out. 2016. doi:http://dx.doi. org/10.17058/cinergis. v17i3.8133. 